\title{
Ventriculectomia parcial esquerda: operação de Batista em pacientes acima de 60 anos
}

\author{
José Carlos D. V. PONTES*, Otoni M. GOMES**,. Carlos Geraldo S. MEDEIROS*, Arino F. SILVA*, \\ João J. DUARTE* ${ }^{*}$ Neimar GARDENAL*, Marcos Douglas Z. VIOLA*
}

RBCCV 44205-526

Pontes J C D V, Gomes O M, Medeiros C G S, Silva A F, Duarte J J, Gardenal N, Viola M D Z - Ventriculectomia parcial esquerda: operação de Batista em pacientes acima de 60 anos. Rev Bras Cir Cardiovasc 2001; 16(1): 20-7.

RESUMO: Fundamentos: A insuficiência cardíaca constitui-se em grave problema de saúde pública. Recente proposta de tratamento cirúrgico para a insuficiência cardíaca terminal é a ventriculectomia parcial esquerda, não havendo publicações específicas sobre seus benefícios em pacientes acima de 60 anos.

Objetivo: Estudar os resultados obtidos com o tratamento da miocardiopatia dilatada em pacientes acima de 60 anos, submetidos à ventriculectomia parcial esquerda.

Método: Entre maio de 1995 e dezembro de $1997 \mathrm{dez}$ pacientes com idade entre 62 e 78 anos, portadores de miocardiopatia dilatada em classe funcional IV (NYHA), foram submetidos à ventriculectomia parcial esquerda no Serviço de Cirurgia Cardiotorácica da Disciplina de Cirurgia Cardiotorácica da Universidade Federal de Mato Grosso do Sul - Núcleo de Hospital Universitário. A ressecção, de forma elíptica, foi realizada na parede lateral do VE, entre os dois músculos papilares, estendendose, desde o ápice até próximo do anel mitral.

Resultados: Nove $(90 \%)$ pacientes receberam alta hospitalar e um paciente $(10 \%)$ evoluiu para óbito, no sexto dia de pós-operatório. O fragmento ressecado variou de 6 a $10,5 \mathrm{~cm}$ de comprimento por 4 a $5,5 \mathrm{~cm}$ de largura, com $10,8 \pm 1,3 \mathrm{~cm}$ por $5 \pm 0,06 \mathrm{~cm}$ em média. O controle ecocardiográfico demonstrou:

\begin{tabular}{llll} 
& Pré-operatório & Pós-operatório & Valor de p \\
\hline FE (\%) & $24,4 \pm 5,56$ & $42,45 \pm 4,57$ & 0,0001 \\
FencS (\%) & $12,3 \pm 3,63$ & $20,80 \pm 2,65$ & 0,000133 \\
DS VE (mm) & $71,0 \pm 5,18$ & $54,55 \pm 5,34$ & 4,25 \\
DD VE (mm) & $80,7 \pm 7,74$ & $69,55 \pm 6,91$ & 0,00191 \\
VDf (ml) & $359,66 \pm 74,11$ & $254,70 \pm 59,10$ & 0,001975 \\
VSf (ml) & $90,22 \pm 40,41$ & $108,28 \pm 28,95$ & 0.33695 \\
\hline
\end{tabular}

No seguimento, observou-se que três pacientes (30\%) encontram-se em grau funcional । ( NYHA), três pacientes $(30 \%)$ em II e um paciente $(10 \%)$ em grau funcional III. A sobrevida atuarial desses pacientes foi de $100 \%, 87 \%, 87 \%$ e $67 \%$ para $6,12,24$ e 36 meses de seguimento, respectivamente.

Conclusão: A ventriculectomia parcial esquerda, realizada em pacientes com idade superior a 60 anos portadores de insuficiência cardíaca com severa disfunção hemodinâmica, apresentou mortalidade baixa, promovendo recuperação acentuada do desempenho cardíaco.

DESCRITORES: Miocardiopatia congestiva, cirurgia. Ventrículo cardíaco, cirurgia. Procedimentos cirúrgicos cardíacos, métodos. Miocardiopatia, cirurgia, idoso. Insuficiência miocárdica, transplante.

\footnotetext{
Trabalho realizado no Serviço de Cirurgia Cardiotorácica do Núcleo de Hospital Universitário da Universidade Federal de Mato Grosso do Sul. Campo Grande, MS, Brasil

Recebido para publicação em novembro de 2000.

* Da Disciplina de Cirurgia Cardiotorácica da Universidade Federal de Mato Grosso do Sul. Campo Grande.

** Da Fundação Cardiovascular São Francisco de Assis - Serviço do Coração, Belo Horizonte.

Endereço para correspondência: José Carlos Dorsa Vieira Pontes. Rua Cândido Mariano, 2581. Campo Grande, MS, Brasil. CEP: $79020-164$.

Tel./Fax.67 784-6180.e-mail: jcarlosdorsa@uol.com.br
} 
Pontes J C D V, Gomes O M, Medeiros C G S, Silva A F, Duarte J J, Gardenal N, Viola M D Z - Ventriculectomia parcial esquerda: operação de Batista em pacientes acima de 60 anos. Rev Bras Cir Cardiovasc 2001; 16(1): 20-7.

\section{INTRODUÇÃO}

O transplante cardíaco tem sido o principal tratamento cirúrgico proposto a pacientes portadores de insuficiência cardíaca irreversível, acompanhada de grave repercussão funcional e hemodinâmica ${ }^{(1,2)}$, determinando mudança expressiva no prognóstico dessa grave enfermidade ${ }^{(3)}$. No entanto, está relacionado a várias limitações, como o número escasso de doadores de órgão, contra-indicações médicas ou psicossociais e os efeitos adversos da terapêutica imunossupressora (4).

Novas modalidades terapêuticas também têm sido propostas no tratamento da insuficiência cardíaca terminal. Esses procedimentos incluem os dispositivos de assistência circulatória mecânica e o coração artificial, que apesar de substituírem totalmente a função circulatória, têm utilização limitada pelos fenômenos tromboembólicos e infecciosos. A despeito de serem já uma realidade, seu elevado custo ainda limita sua utilização $(5,6)$.

Outra proposição de tratamento cirúrgico para as cardiopatias terminais constitui-se nos enxertos musculares esqueléticos pediculados, estimulados em sincronia com o coração, passando dessa forma a auxiliar a contração ventricular, recebendo a denominação de cardiomioplastia (7-9)

BATISTA et al. (10), em 1997, apresentaram casuística de 120 pacientes em estágio final de miocardiopatia dilatada por diversas causas, onde uma nova opção cirúrgica denominada ventriculectomia parcial esquerda foi empregada, observando-se $22 \%$ de mortalidade hospitalar e $55 \%$ de sobrevida atuarial em dois anos de seguimento.

Os resultados favoráveis obtidos no tratamento da insuficiência cardíaca refratária com a operação de Batista, no Hospital Universitário da Universidade Federal de Mato Grosso do Sul (11-13), motivaram a extensão de sua indicação em faixa etária não avaliada especificamente na literatura acessível, constituindo dessa forma o objetivo da presente investigação a análise dos resultados obtidos com o tratamento da miocardiopatia dilatada, em 10 pacientes com idade superior a 60 anos, submetidos à ventriculectomia parcial esquerda.

\section{MÉTODO}

No Serviço de Cirurgia Cardiotorácica da Disciplina de Cirurgia Cardiotorácica do Departamento de Clínica Cirúrgica do Centro de Ciências Biológicas e da Saúde da Universidade Federal de Mato Grosso do Sul - Núcleo de Hospital Universitário, entre maio de 1995 e janeiro de 1998, 30 pacientes portadores de insuficiência cardíaca terminal foram submetidos à ventriculectomia parcial esquerda (Operação de Batista). Uma série de 10 pacientes, em classe funcional IV de acordo com o Criteria Commitee of The New York Heart Association (14), com idades entre 62 e 78 anos, foi selecionada para este estudo, sendo 6 pacientes $(60 \%)$ do sexo masculino e $4(40 \%)$ do sexo feminino.

Todos os pacientes encontravam-se em tratamento medicamentoso máximo admissível de drogas e com registro de pelo menos duas internações nos últimos três meses, que precederam a indicação da cirurgia, devido à insuficiência cardíaca descompensada. Todos eram portadores de edema crônico em membros inferires, com restrição à deambulação.

Nos exames complementares apresentavam ao ecocardiograma fração de ejeção média de 24,40 \pm $06,56 \%$ e diâmetro diastólico do ventrículo esquerdo médio de $80,70 \pm 07,74 \mathrm{~mm}$ e ao estudo hemodinâmico, apresentavam pressão diastólica final do ventrículo esquerdo $\left(\mathrm{Pd}_{2}\right.$ de $\left.\mathrm{VE}\right)$ superior a $18 \mathrm{mmHg}$.

Etiologicamente, 9 pacientes eram portadores de cardiomiopatia dilatada e 1 de miocardiopatia chagásica. Todos apresentavam insuficiência mitral secundária à dilatação das cavidades esquerdas e hipocontratilidade severa do ventrículo esquerdo.

Os pacientes foram operados em circulação extracorpórea, utilizando-se bomba de roletes e oxigenadores de membranas de fibras ocas com filtro de linha arterial. As perfusões foram realizadas em hipotermia moderada $\left(32^{\circ} \mathrm{C}\right)$ e hemodiluição total. Como proteção miocárdica manteve-se a perfusão coronária, sendo a aorta pinçada apenas por períodos máximos de cinco minutos, para aliviar a tensão no final da ventriculorrafia e no momento da abertura do átrio esquerdo, até a introdução de cânula multiperfurada no ventrículo esquerdo, através do orifício mitral. Durante os curtos períodos de pinçamento aórtico, as câmaras esquerdas foram descomprimidas por aspiração da raiz aórtica por meio de cânula plástica 14 G (Gauge).

Com a aorta desclampeada, o apex cordis foi aberto, ressecando-se seguimento elíptico do ventrículo esquerdo, correspondente à musculatura entre os músculos papilares da valva mitral, tomando-se o cuidado de não atingir o sulco atrioventricular. Os ramos coronários seccionados foram cauterizados e em seguida realizou-se ventriculorrafia com duplo plano em chuleio contínuo, com fio de polipropileno 000 . Os espécimes cirúrgicos tiveram peso entre 30 e $60 \mathrm{~g}$, aferidos em balança eletrônica de precisão, sendo enviados para estudo anatomopatológico. 
Pontes J C D V, Gomes O M, Medeiros C G S, Silva A F, Duarte J J, Gardenal N, Viola M D Z - Ventriculectomia parcial esquerda: operação de Batista em pacientes acima de 60 anos. Rev Bras Cir Cardiovasc 2001; 16(1): 20-7.

A deaeração das cavidades esquerdas, nesse momento, foi realizada através da ventriculotomia, com a aorta pinçada, antes da finalização da ventriculorrafia.

Em seguida, o átrio esquerdo foi aberto paralelamente ao septo interatrial com a aorta pinçada, até a introdução de cânula plástica (3/8) multiperfurada, através do orifício mitral, com o intuito de se evitar a embolia aérea. Com a aorta despinçada e mantendo-se a cânula multiperfurada para preservar a valva mitral aberta, realizou-se anuloplastia mitral, por meio da passagem de 6 a 8 fios de poliéster 00 em figura de "U", que foram ancorados posteriormente em barra de pericárdio bovino, dimensionada em função da cúspide anterior da valva mitral. O teste da suficiência valvar foi realizado com o coração batendo em todos os casos. Em seguida, mantendo-se a valva mitral insuficiente por meio da mesma cânula plástica multiperfurada, com intuito de se evitar embolia aérea, enquanto com o coração batendo em normotermia, procedia-se à atriorrafia, através de chuleio contínuo em plano único com polipropileno 000. Seguiu-se, ainda em extracorpórea, criteriosa revisão da hemostasia do ventrículo esquerdo.

Os tempos de observação e acompanhamento variaram de 6 a 36 meses.

A avaliação pós-operatória foi realizada considerando-se a classe funcional da insuficiência cardíaca, eletrocardiograma, radiografia do tórax e ecocardiograma, no período hospitalar, após o primeiro mês e no seguimento tardio, conforme o tempo de evolução de cada caso.

\section{Estudo Estatístico}

Foi realizado o Teste "t de Student" para análise pareada, com nível de significância em 0,05 (15) e curva atuarial de sobrevida (16).

\section{RESULTADOS}

Nove pacientes (90\%) receberam alta hospitalar, em condições clínicas estáveis.

O tempo médio de cirurgia foi de $3,75+0,63$ horas com $45 \pm 8,49$ minutos de circulação extracorpórea. $O$ fragmento ventricular ressecado variou de 6 a $10,5 \mathrm{~cm}$ de comprimento por 4 a 5,5 $\mathrm{cm}$ de largura, com $10,8 \pm 1,3 \mathrm{~cm}$ por $5 \pm 0,06 \mathrm{~cm}$ na média, pesando $41 \pm 15 \mathrm{~g}$ em média. O estudo histológico revelou hipertrofia dos miócitos em todos $(100 \%)$, edema intersticial em 4 (40\%), fibrose intersticial em $6(60 \%)$ e infiltrado mononuclear em 1 (10\%).
Após saída de CEC, 50\% apresentavam-se em ritmo sinusal, $20 \%$ em fibrilação atrial, $10 \%$ em ritmo juncional e $20 \%$ em bloqueio atrioventricular total (BAVT), sendo necessário implante de marcapasso definitivo em um caso.

No pós-operatório imediato, observou-se tempo médio para extubação de $6,6 \pm 5,18$ horas. A alta da unidade intensiva ocorreu no terceiro dia de pósoperatório em $50 \%$ dos casos, no segundo dia em $10 \%$, quarto dia em $10 \%$, quinto dia em $10 \%$ e sexto dia de pós-operatório em $10 \%$ dos casos. Dois pacientes evoluíram com insuficiência renal aguda, sendo um deles tratado clinicamente, com boa resolução e outro, tratado com diálise peritoneal, que, apesar da melhora com a terapêutica dialítica, evoluiu para óbito no sexto dia de pós-operatório, por insuficiência respiratória aguda .

$\mathrm{Na}$ fase hospitalar, não houve ocorrência de trombose nem embolia pulmonar e o tempo médio de internação foi de 12,6 dias.

O controle ecocardiográfico apresentado na Tabela 1 comprovou melhora global da função ventricular esquerda.

O comportamento da fração de ejeção, assim como o diâmetro diastólico do ventrículo esquerdo, avaliados pela ecocardiografia ao longo dos meses de seguimento pós-operatório, apresentouse estável.

A última avaliação clínica pós-operatória demonstrou que 3 pacientes (30\%) encontram-se em grau funcional I (NYHA), 3 pacientes $(30 \%)$ em grau funcional II e 1 paciente (10\%) em grau III. Os casos nos 3 e 8 evoluíram para óbito aos 28 e 4 meses de seguimento, devido a progressão da insuficiência cardíaca e morte súbita, respectivamente.

A sobrevida atuarial desses pacientes, considerando-se pacientes vivos após 30 dias da cirurgia, foi de $100 \%, 87 \%, 87 \%$ e $67 \%$ para $6,12,24$ e 36 meses de seguimento, respectivamente (Figura 1).

\section{DISCUSSÃO}

Recente proposta de tratamento cirúrgico para a insuficiência cardíaca terminal tem sido a ventriculectomia parcial esquerda proposta por BATISTA et al. (17), na qual a ressecção de uma elipse na parede ventircular determina diminuição do raio da cavidade ventricular, produzindo adequação na relação volume/massa e, dessa forma, baseando-se na lei de La Place, interfere no trabalho cardíaco, por diminuir a tensão parietal, reduzindo o consumo de oxigênio do miocárdio. BATISTA et al. (18), em 103 pacientes operados de 1974 a 1995, 
Pontes J C D V, Gomes O M, Medeiros C G S, Silva A F, Duarte J J, Gardenal N, Viola M D Z - Ventriculectomia parcial esquerda: operação de Batista em pacientes acima de 60 anos. Rev Bras Cir Cardiovasc 2001; 16(1): 20-7.

TABELA 1

\begin{tabular}{lccc}
\hline & \multicolumn{2}{c}{ DADOS DA AVALIAÇÃO ECOCARDIOGRÁFICA COMPARANDO-SE PRÉ E PÓS-OPERATORIO } \\
\hline & PRÉ-OPERATÓRIO & PÓS-OPERATÓRIO & VALOR DE p \\
\hline FE (\%) & $24,4 \pm 5,56$ & $42,45 \pm 4,57$ & 0,0001 \\
EncS (\%) & $12,3 \pm 3,63$ & $20,80 \pm 2,65$ & 0,000133 \\
DS VE (mm) & $71,0 \pm 5,18$ & $54,55 \pm 5,34$ & 4,25 \\
DD VE (mm) & $80,7 \pm 7,74$ & $69,55 \pm 6,91$ & 0,00191 \\
VDf (ml) & $359,66 \pm 74,11$ & $254,70 \pm 59,10$ & 0,001975 \\
VSf (ml) & $90,22 \pm 40,41$ & $108,28 \pm 28,95$ & 0.33695
\end{tabular}

$\mathrm{FE}=$ fração de ejeção. Enc $=$ Percentual de encurtamento sistólico. DSVE $=$ diâmetro sistólico do ventrículo esquerdo. DDVE $=$ diâmetro diastólico do ventrículo esquerdo. $\mathrm{VDf}=$ volume diastólico final. $\mathrm{VSf}=$ volume sistólico final.

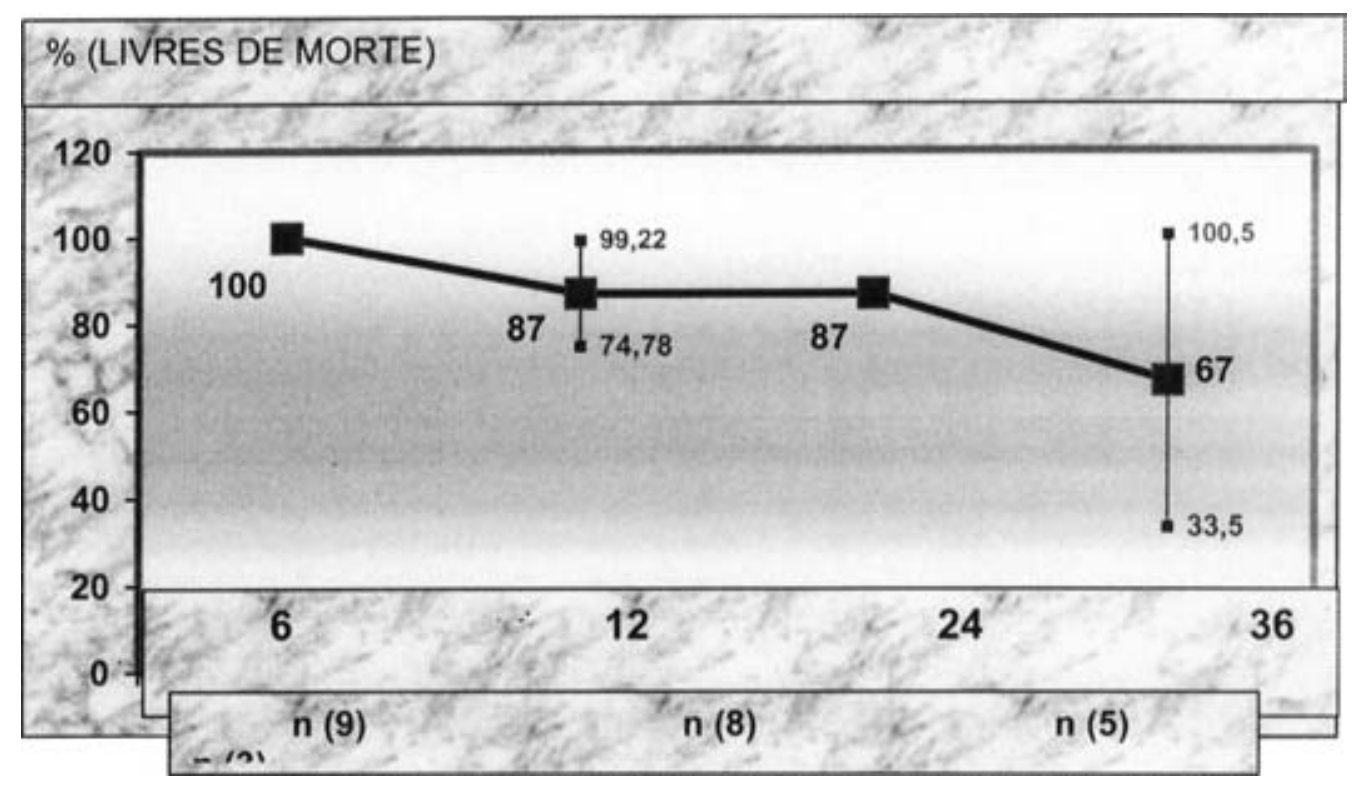

Fig. 1 - Curva atuarial de sobrevida de pacientes com idade superior a 60 anos em classe funcional IV de insuficiência cardíaca (NYHA), submetidos à ventriculectomia parcial esquerda com seguimento de até três anos com os respectivos cohortes por intervalo de erros padrões.

obtiveram mortalidade hospitalar de $13 \%$, com melhora substancial na função sistólica do ventrículo esquerdo, resultados confirmados em vários centros de cirurgia cardíaca.

BELLOTTI et al. (19), em 1996, confirmaram a proposição de Batista, relatando que a ventriculectomia parcial esquerda produziu importantes e significativas modificações nos parâmetros de função sistólica, de contratilidade, de mecânica, de forma e de geometria do ventrículo esquerdo.

FROTA FILHO et al. (20), em 1996, BOCCHI et al. (21), em 1997, McCARTHY et al. (22), em 1997,
MOREIRA et al. (23), em 1997, relataram casuísticas onde obtiveram significativa redução nos diâmetros do ventrículo esquerdo e importante incremento na fração de ejeção.

$\mathrm{Na}$ presente investigação, considerando-se pacientes com idade superior a 60 anos com insuficiência cardíaca classe funcional IV, submetidos à ventriculectomia parcial esquerda, a fração de ejeção de $24,40 \pm 6,56 \%$, em média, no pré-operatório, passou para $42,45 \pm 4,57 \%$, em média, após o primeiro mês de pós-operatório, de maneira estatisticamente significativa $(p<0,05)$, mantendo-se estável em até três anos de seguimento, semelhante 
Pontes J C D V, Gomes O M, Medeiros C G S, Silva A F, Duarte J J, Gardenal N, Viola M D Z - Ventriculectomia parcial esquerda: operação de Batista em pacientes acima de 60 anos. Rev Bras Cir Cardiovasc 2001; 16(1): 20-7.

aos resultados apresentados por MOREIRA et al. (24), em 1998. Ainda nesta casuística de pacientes sexagenários, ocorreu redução do diâmetro diastólico e sistólico, com incremento significativo da porcentagem de encurtamento sistólico do ventrículo esquerdo, assim como a adequada relação volume/ massa foi restabelecida.

A análise da evolução da classe funcional demonstrou que $80 \%$ dos pacientes, após seis meses de acompanhamento, apresentavam-se livres de classe funcional III ou IV.

A insuficiência cardíaca em classe funcional IV, estágio evolutivo em que se encontravam os pacientes sexagenários desta pesquisa no período préoperatório, possui prognóstico reservado quando adotado somente medidas terapêuticas clínicas ${ }^{(26)}$.

Vários estudos (25-27) sobre mortalidade na insuficiência cardíaca avançada demonstram perspectivas sombrias em poucos meses de seguimento, quando se adota apenas a terapêutica medicamentosa. No grupo etário de pacientes com idade superior a 60 anos, ocorre aumento proporcional da mortalidade com o envelhecimento (28). Admite-se, portanto, que, numa faixa etária mais avançada, a insuficiência cardíaca pode apresentar-se com prognóstico ainda pior. Diante do exposto, o tratamento cirúrgico se impõe para esse grupo de enfermos na tentativa de melhorar a qualidade de vida e sobrevida.

O transplante cardíaco tem sido a principal terapêutica para a insuficiência cardíaca terminal, porém, em pacientes com idade superior a 60 anos, relaciona-se a maior incidência de diabete induzido por corticosteróides, osteoporose e infecções graves como relataram OLIVARI et al. (29). Dessa maneira, os pacientes acima de 60 anos de idade geralmente perdem a prioridade nas filas de transplante cardíaco, dadas as relativas contra-indicações, face aos menores benefícios obtidos, em relação aos pacientes mais jovens, apesar de apresentarem sobrevida atuarial semelhante, em seguimento de cinco anos (30).

Considerando-se a possibilidade do tratamento clínico para esses graves enfermos, utilizando-se drogas de última geração atuantes na resposta neuroendócrica do organismo à insuficiência cardíaca destacamos os beta-bloquedores e os inibidores da enzima conversora do angiotensinogênio. Esses fármacos são referidos por PARKER (31), HALL (32) e no Estudo Consensus (25) como drogas capazes de melhorar significativamente os sintomas da falência cardíaca assim como interferir na sobrevida dos pacientes. Esses autores $^{(25,31,32)}$ relataram sobrevida de $53 \%, 37 \%$, e $20 \%$ para um, dois e três anos de acompanhamento, respectivamente.
Considerando-se ainda os problemas inerentes ao tratamento da insuficiência cardíaca no idoso e à realização de procedimentos cirúrgicos de grande porte nessa faixa etária, destaca-se no presente estudo a relativa baixa incidência de morbimortalidade, semelhante ao descrito para cirurgias cardíacas convencionais em pacientes acima de 60 anos de idade (3-36). Nesta investigação, constatouse $10 \%$ de mortalidade hospitalar e sobrevida atuarial de $100 \%, 87 \%, 87 \%$ e $67 \%$ para $6,12,24$ e 36 meses de seguimento, respectivamente, demonstrando que foi possível, por meio da ventriculectomia parcial esquerda, mudar a história natural dessa grave afecção, mesmo comparandose às curvas de sobrevivência de pacientes tratados com drogas de última geração no tratamento da insuficiência cardíaca $(25,31,32)$. Os resultados da presente investigação foram superponíveis às estatísticas de sobrevida semestral apresentadas por outros autores (10,22,24,37-39) para a Operação de Batista, realizada sem considerar faixa etária específica, divergindo porém dos resultados insatisfatórios apresentados por MORAES et al. ${ }^{(40)}$, que apresentaram mortalidade global de $24 \%$, FROTA FILHO et al. (41) com mortalidade imediata de $21,5 \%$ e sobrevida atuarial de 36,6\% em 24 meses de seguimento e BRASIL et al. (42), com mortalidade hospitalar de $37 \%$.

Em análise geral, os resultados obtidos nesta investigação permitem concluir que a ventriculectomia parcial esquerda pode ser realizada, em pacientes com idade acima de 60 anos, com aceitável morbimortalidade em presença de insuficiência cardíaca severa.

\section{CONCLUSÕES}

A ventriculectomia parcial esquerda possibilitou o tratamento de pacientes com idade superior a 60 anos, portadores de insuficiência cardíaca com severa disfunção hemodinâmica, com baixa mortalidade hospitalar e boa sobrevida atuarial até 36 meses de seguimento.

- A Operação de Batista, realizada em pacientes com idade superior a 60 anos, promoveu recuperação acentuada do desempenho cardíaco, evidenciado por: melhora da classe funcional (NYHA); aumento da fração de ejeção e porcentual de encurtamento sistólico do ventrículo esquerdo; diminuição dos diâmetros sistólico e diastólico do ventrículo esquerdo e por ter determinando melhor adequação da relação volume/massa do ventrículo esquerdo. 
Pontes J C D V, Gomes O M, Medeiros C G S, Silva A F, Duarte J J, Gardenal N, Viola M D Z - Ventriculectomia parcial esquerda: operação de Batista em pacientes acima de 60 anos. Rev Bras Cir Cardiovasc 2001; 16(1): 20-7.

RBCCV 44205-526

Pontes J C D V, Gomes O M, Medeiros C G S, Silva A F, Duarte J J, Gardenal N, Viola M D Z - Partial left ventriculectomy: Batista's Operation In elderly patients. Rev Bras Cir Cardiovasc 2001; 16(1): 20-7.

ABSTRACT: Background: Cardiac insufficiency is one of the most important problems challenging the public health resources in the modern world. The partial left ventriculectomy (Batista's Operation) is the most recent surgical treatment for the end-stage heart failure but, until now, without specific reports in the cardiovascular literature regarding its results in elderly patients.

Objective: To study the results of the Batista's Operation in aged patients.

Method: From May 1995 to December 1997, ten patients aged 62 to 78 years, suffering from dilated cardiomyopathy (class IV NYHA), were treated with the partial left ventriculectomy procedure in the Cardiothoracic Surgical Clinic of the Cardiothoracic Surgery Department of Mato Grosso do Sul Federal University - (University Hospital Nucleus).

Results: Nine (90\%) patients were discharged from the hospital and one (10\%) patient died in the $6^{\text {th }}$ postoperative day from respiratory insufficiency. An elliptical resection was performed in the lateral wall of the left ventricle, from the apex to near the atrioventricular groove and between the papillary muscles. Dimensions of the removed fragment (mean values): $10.8 \pm 1.3 \mathrm{~cm} \times 5.0 \pm 0.6 \mathrm{~cm}$. The echocardiographic control has proved:

\begin{tabular}{lccl}
\hline & Pre-operative period & Postoperative period & \multicolumn{1}{c}{$\mathbf{p =}$} \\
\hline EF $(\%)$ & $24.4 \pm 5.56$ & $42.45 \pm 4.57$ & 0.0001 \\
FE $(\%)$ & $12.3 \pm 3.63$ & $20.80 \pm 2.65$ & 0.000133 \\
LVSD (mm) & $71.0 \pm 5.18$ & $54.55 \pm 5.34$ & 4.25 \\
LVDD (mm) & $80.7 \pm 7.74$ & $69.55 \pm 6.91$ & 0.00191 \\
DVL $(\mathrm{ml})$ & $359.66 \pm 74.11$ & $254.70 \pm 59.10$ & 0.001975 \\
SVR $(\mathrm{ml})$ & $90.22 \pm 40.41$ & $108.28 \pm 28.95$ & 0.33695 \\
\hline
\end{tabular}

$\mathrm{FE}=$ fractional shortening, $\mathrm{EF}=$ ejection fraction, $\mathrm{LVSD}=$ left ventricular end-systolic diameter, LVDD = left ventricular end-diastolic diameter, DVL = diastolic volume lowering and SVR = systolic volume rised. In the late follow up three patients were in NYHA class I, three patients in class II and one in class III. The actuarial survival rate were $100 \%, 87 \%, 87 \%$ and $67 \%$ for $6,12,24$ and 36 months respectively.

Conclusion: The partial left ventriculectomy performed in elderly patients with cardiac insufficiency with severe hemodynamics disfunction resulted in 10\% mortality, promoted important recovery of the cardiac performance by: improvement of NYHA functional class, enhancement of the ejection fraction and left ventricular \% systolic shortening; reduction of the left ventricular systolic and diastolic diameters and determining better ventricular volume/mass ratio.

DESCRIPTORS: Cardiomyopathy, congestive, sugery. Heart ventricle, sugery. Cardiomyopathy, surgery, aged. Myocardial insufficiency, transplantation.

\section{REFRÊNCIAS BIBLIOGRÁFICAS}

1 Moreira L F, Stolf N A G, Jatene A D - Alternativas cirúrgicas do transplante. In: 25 anos de transplante cardíaco no Brasil. Departamento Científico do Centro Acadêmico Oswaldo Cruz da Faculdade de Medicina da Universidade de São Paulo,1992.

2 Stolf N A G \& Jatene A D - História do transplante cardíaco. Rev Soc Cardiol Estado de São Paulo 1995; 5: 609-13.

3 Hosenpud J D, Novick R J, Breen T J, Daily O P Registry of the International Society for Heart and Lung Transplantation: Eleventh Official Report. J Heart Lung Transplant 1994; 13: 561-70.

4 Kirklin J K, Naftel D C, Bourge R C et al. - Rejection after cardiac transplantation: a time-related risk factor analysis. Circulation 1992; 86 (5 Suppl): II 236-41.

5 McCarthy P M, Schimitt S K, Vargo R L, Gordon S, Keys T F, Hobbs R E - Implantable LVAD infections: implications for permanent use of the device. Ann Thorac Surg; 1996; 61: 359-73.

6 Frazier O H - The development, evolution, and clinical utilization of artificial heart technology. Eur $J$ Cardiothorac Surg; 1997; 11 (Suppl): S29-31.

7 Kantrowitz A \& Mckinnon W - The experimental use of the diaphragm as an auxiliary myocardium. Surg Forum 1959; 9: 266-8.

8 Hune W I - Construction of a functioning accessory myocardium. Trans South Surg Assoc 1968; 79:200-2. 
Pontes J C D V, Gomes O M, Medeiros C G S, Silva A F, Duarte J J, Gardenal N, Viola M D Z - Ventriculectomia parcial esquerda: operação de Batista em pacientes acima de 60 anos. Rev Bras Cir Cardiovasc 2001; 16(1): 20-7.

9 Carpentier A \& Chachques J C - Myocardial substitution with a skeletal muscle: first successful clinical case (Letter). Lancet 1985; 1: 1267.

10 Batista R J, Verde J, Nery P et al. - Partial left ventriculectomy to treat end-stage heart disease. Ann Thorac Surg 1997; 64: 634-8.

11 Pontes J C D, Medeiros C G S, Porto A S et al. Ventriculectomia parcial: experiência inicial no Serviço de Cirurgia Cardiotorácica do Núcleo de Hospital Universitário da Universidade Federal de Mato Grosso do Sul. In : Forum Científico V, Belo Horizonte, 1995

12 Pontes J C D, Medeiros C G S, Porto A S et al. Ventriculectomia parcial: experiência inicial no Serviço de Cirurgia Cardiotorácica do Núcleo de Hospital Universitário da Universidade Federal de Mato Grosso do Sul. Coração 1995; 5: 6.

13 Pontes J C D, Medeiros C G S, Silva A F et al. Ventriculectomia parcial: nova técnica para tratar a insuficiência cardíaca em fase terminal. Relato da experiência inicial no Serviço de Cirurgia Cardiotorácica do Núcleo de Hospital Universitário da Universidade Federal de Mato Grosso do Sul. In: Forum Científico VI, Belo Horizonte, 1996.

14 Criteria Committee, New York Heart Association, Inc.: Diseases of the heart and blood vessels. Nomenclature and criteria for diagnosis. 6. ed. Boston, Little, Brown, 1964:114.

15 Snedecor W G \& Cochran W C - The comparison of two samples. In : Statistical methods. 7. ed. AMES; The lowa State University Press, 1980: 83.

16 Braile D M - Noções de estatística aplicada à cirurgia cardíaca. Apostila Braile Biomédica. 1997; 54p.

17 Batista R J V, Santos J L V, Cunha M A et al. Ventriculectomia parcial: um novo conceito no tratamento cirúrgico de cardiopatias em fase terminal. In: Anais do XXII Congresso Nacional de Cirurgia Cardíaca. Brasília, Sociedade Brasileira de Cirurgia Cardiovascular, 1995:150-1.

18 Batista R J V, Santos J L V, Franzoni M et al. Ventriculectomia parcial: um novo conceito no tratamento cirúrgico de cardiopatias em fase final. Rev Bras Cir Cardiovas 1996; 11: 1-6.

19 Bellotti G, Moraes A, Bocchi E et al. - Efeitos da ventriculectomia parcial nas propriedades mecânicas, forma e geometria do ventrículo esquerdo em portadores de cardiomiopatia dilatada. Arq Bras Cardiol 1996; 67: 395-400.

20 Frota Filho J D, Pereira W M, Leães P E, Blancher C, Jung $L A$, Lucchese $F$ - End-stage heart failure: is there a role for the Batista procedure? Heart Surg Forum, 1996; 1: 41-8.

21 Bocchi E A, Bellotti G, Vilella de Moraes A et al. -
Clinical outcome after left ventricular surgical remodeling in patients with idiopathic dilated cardiomyopathy referred for heart transplantation: short-term results. Circulation 1997; 96 (9 Suppl): II165-72.

22 McCarthy $\mathrm{P}$ M, Starling R C, Wong J et al. - Early results with partial left ventriculectomy. J Thorac Cardiovasc Surg 1997; 114: 755-65.

23 Moreira L F P, Stolf N A G, Bocchi E A, Bacal F, Bellotti $G$, Jatene A D - Análise comparativa dos resultados da cardiomioplastia e da ventriculectomia parcial no tratamento da cardiomiopatia dilatada. In: Congresso Brasileiro de Cardiologia. São Paulo, SP: Sociedade Brasileira de Cardiologia, 1997.

24 Moreira L F, Stolf N A G, Bocchi E A et al. - Existe lugar para a ventriculectomia parcial esquerda no tratamento da cardiomiopatia dilatada? In: $25^{\circ}$ Congresso Nacional de Cirurgia Cardíaca. São Paulo, SP: Sociedade Brasileira de Cirurgia Cardiovascular, 1998.

25 The Consensus Trial Study Group : Effects of enalapril on mortality in severe congestive heart failure. $N$ Engl J Med 1997; 316: 1429-35.

26 Moreira L F, Stolf N A G, Jatene A D - Alternativas cirúrgicas do transplante. In : 25 anos de transplante cardíaco no Brasil. Departamento Científico do Centro Acadêmico Oswaldo Cruz da Faculdade de Medicina da Universidade de São Paulo, 1992.

27 Moreira L F P, Stolf N A G, Bocchi E A et al. - Resultados da cardiomioplastia no tratamento da cardiomiopatia dilatada. Rev Bras Cir Cardiovasc 1991; 6: 85-95

28 Yazaki L M \& Saad P M - Mortalidade da população idosa. In: Coleção Realidade Paulista, SEAD eds. O idoso na grande São Paulo. São Paulo: SP, 1990.

29 Olivari M T, Atolick A, Kaye M P - Heart transplantation in elderly patients. J Heart Transplant 1988; 7: 258-64.

30 Aravot D T, Banner N R, Khagani A - Cardiac transplantation in the seventh decade of life. $A m \mathrm{~J}$ Cardiol 1989; 63: 90-3.

31 Parker M - Treatment of chronic heart failure. Lancet 1992. 340:92 - J (Review).

32 Hall S A Cigarroa C G, Morreoux L, Risser R C, Gryburn $P$ A, Eichburn $E J$ - Time course of omprovement in left ventricular function, mass and geometry in patients with congestive heart failure treated with beta-adrenergic blockade. J Am Coll Cardiol 1995; 25: 54-61.

33 Silva L H F, Nascimento C S, Viotti Jr. L A P Revascularização do miocádio em idosos. Rev Bras Cir Cardiovasc 1997; 12: 132-40.

34 Loop F D, Lytle B W, Cosgrove D M et al. - Coronary artery bypass graft surgery in elderly. Clive $\mathrm{J}$ Med 1988; 55: 23-4. 
Pontes J C D V, Gomes O M, Medeiros C G S, Silva A F, Duarte J J, Gardenal N, Viola M D Z - Ventriculectomia parcial esquerda: operação de Batista em pacientes acima de 60 anos. Rev Bras Cir Cardiovasc 2001; 16(1): 20-7.

35 Mikaeloff O J - La chirurgie cardiaque du sujet âgé. Presse Med 1991; 20: 589-92.

36 Iglézias J C R, Dallan L A, Oliveira S A et al. Revascularização do miocárdio no paciente octagenário: 15 anos de observação. Rev Bras Cir Cardiovasc 1993; 8: 237-40.

37 Bombonato R, Bestetti R B, Sgarbieri R et al. - Experiência inicial com a ventriculectomia parcial esquerda no tratamento da insuficiência cardíaca terminal. Arq Bras Cardiol 1996; 66: 189-92.

38 Anelini G D, Pryn S, Mehta D et al. - Left-ventricularvolume reductionfor end-stage heart failure. Lancet 1997; 350: 489.(Letter)

39 Vijayanagar R, Weston $\mathrm{M}$, Sears $\mathrm{N}$ et al. - Partial left ventriculectomy ( $\mathrm{plv}$ ) for treatment of end-stage heart disease (eshd): evaluation of early experience. Am College Chest Physicians 1997; 112: 3S.

40 Moraes J B M, Carvalho H P, Ferreira E S et al. Ventriculectomia esquerda parcial redutora. In: Congresso Brasileiro de Cardiologia, São Paulo, SP: Sociedade Brasileira de Cardiologia, 1997. (Resumos).

41 Frota Filho J D, Lucchese F, Blacher C et al. - Três anos de ventriculectomia parcial esquerda: resultados globais e tardios em 41 pacientes. Rev Bras Cir Cardiovasc, 1999; 14: 75-87.

42 Brasil J C F, Bombonato R, Moreira Neto F F, Sgarbieri R N, Munhoz N C - Ventriculectomia parcial esquerda: experiência inicial. In: $25^{\circ}$ Congresso Nacional de Cirurgia Cardíaca. São Paulo, SP: Sociedade Brasileira de Cirurgia Cardiovascular, 1998. (Resumos). 